\title{
ALGEBRAIC INDEPENDENCE RESULTS FOR VALUES OF THETA-CONSTANTS
}

Carsten Elsner

Abstract: Let $\theta(q)=1+2 \sum_{\nu=1}^{\infty} q^{\nu^{2}}$ denote the Thetanullwert of the Jacobi Zeta function

$$
\theta(z \mid \tau)=\sum_{\nu=-\infty}^{\infty} e^{\pi i \nu^{2} \tau+2 \pi i \nu z}
$$

For algebraic numbers $q$ with $0<|q|<1$ we prove the algebraic independence over $\mathbb{Q}$ of the numbers $\theta\left(q^{n}\right)$ and $\theta(q)$ for $n=2,3, \ldots, 12$ and furthermore for all $n \geqslant 16$ which are powers of two. An application for $n=5$ proves the transcendence of the number

$$
\sum_{j=1}^{\infty}(-1)^{j}\left(\frac{j}{5}\right) \frac{j q^{j}}{1-q^{j}}
$$

Similar results are obtained for numbers related to modular equations of degree 3, 5, and 7 .

Keywords: algebraic independence, theta-constants, Nesterenko's theorem, independence criterion, modular equations.

\section{Introduction and statement of results}

Let $\tau$ with $\Im(\tau)>0$ denote a complex variable. The series

$$
\vartheta_{2}(\tau)=2 \sum_{\nu=0}^{\infty} q^{(\nu+1 / 2)^{2}}, \quad \vartheta_{3}(\tau)=1+2 \sum_{\nu=1}^{\infty} q^{\nu^{2}}, \quad \vartheta_{4}(\tau)=1+2 \sum_{\nu=1}^{\infty}(-1)^{\nu} q^{\nu^{2}}
$$

are known as theta-constants or Thetanullwerte, where $q=e^{\pi i \tau}$. Sometimes it is useful to write $\vartheta_{2}(q), \vartheta_{3}(q), \vartheta_{4}(q)$ instead of $\vartheta_{2}(\tau), \vartheta_{3}(\tau), \vartheta_{4}(\tau)$, respectively, where $q$ belongs to the unit circle around 0 of the complex plane. The thetaconstants are modular forms of weight $1 / 2$ for the principal congruence subgroup of level 2. In particular, $\theta(q):=\vartheta_{3}(q)$ is the Thetanullwert of the Jacobi zeta

2010 Mathematics Subject Classification: primary: 11J85; secondary: 11J91, 11F27 
function $\theta(z \mid \tau)=\sum_{\nu=-\infty}^{\infty} e^{\pi i \nu^{2} \tau+2 \pi i \nu z}$. Let $n \geqslant 3$ denote an odd positive integer. Set

$$
h_{j}(\tau):=n^{2} \frac{\vartheta_{j}^{4}(n \tau)}{\vartheta_{j}^{4}(\tau)} \quad(j=2,3,4), \quad \lambda=\lambda(\tau):=\frac{\vartheta_{2}^{4}(\tau)}{\vartheta_{3}^{4}(\tau)}, \quad \psi(n):=n \prod_{p \mid n}\left(1+\frac{1}{p}\right),
$$

where $p$ runs through all primes dividing $n$. Also the function

$$
j(\tau):=256 \frac{\left(\lambda^{2}-\lambda+1\right)^{3}}{\lambda^{2}(\lambda-1)^{2}}
$$

is a modular function with respect to the group $S L(2, \mathbb{Z})$ (cf. [5, ch.3,18]), for which identities of the form $\Phi_{n}(j(\tau), j(n \tau))$ with polynomials $\Phi_{n}(X, Y) \in \mathbb{Z}[X, Y]$ are known (cf. [5, ch.5]). Yu.V.Nesterenko [8] proved the existence of integer polynomials $P_{n}(X, Y) \in \mathbb{Z}[X, Y]$ such that $P_{n}\left(h_{j}(\tau), R_{j}(\lambda(\tau))\right)=0$ holds for $j=2,3,4$, odd integers $n \geqslant 3$, and a suitable rational function $R_{2}, R_{3}$, or $R_{4}$, respectively:

Theorem A ([8, Theorem 1.1, Corollary 3$])$. For any odd integer $n \geqslant 3$ there exists a polynomial $P_{n}(X, Y) \in \mathbb{Z}[X, Y] \operatorname{deg}_{X} P=\psi(n)$, such that

$$
\begin{aligned}
P_{n}\left(h_{2}(\tau), 16 \frac{\lambda(\tau)-1}{\lambda(\tau)}\right) & =0, \\
P_{n}\left(h_{3}(\tau), 16 \lambda(\tau)\right) & =0, \\
P_{n}\left(h_{4}(\tau), 16 \frac{\lambda(\tau)}{\lambda(\tau)-1}\right) & =0 .
\end{aligned}
$$

The polynomials $P_{3}, P_{5}, P_{7}, P_{9}$, and $P_{11}$ are listed in the appendix. $P_{3}$ and $P_{5}$ are already given in [8], $P_{7}, P_{9}$, and $P_{11}$ are the results of computer-assisted computations of the author.

There are various algebraic relationships between the theta-constants and arithmetic functions like Ramanujan's Eisenstein series $P(q), Q(q), R(q)$ (cf. [6]), the Dedekind eta-function $\eta(\tau)=e^{\pi i \tau / 12} \prod_{n=1}^{\infty}\left(1-e^{2 \pi i \tau n}\right)$, and others. For instance, it follows from Jacobi's triple product identity that $\theta(-q)=\eta^{2}(\tau) / \eta(2 \tau)$ for $\Im(\tau)>0$ and $q=e^{2 \pi i \tau}$. Therefore, under suitable circumstances, an algebraic independence result for values of theta-constants can be transformed into an algebraic independence result for functions which are expressed in terms of theta-constants. For example, see [3] and Corollary1.1 below.

In this paper we focus on the problem to decide on the algebraic independence of $\theta(q)$ and $\theta\left(q^{n}\right)$ over $\mathbb{Q}$ for algebraic numbers $q$ and integers $n>1$. We shall use Theorem A in connection with an algebraic independence criterion (Lemma 2.1) to settle the problem for the odd integers $n=3,5,7,9,11$ and for three even numbers $n=6, n=10$, and $n=12$. The central point of the algebraic independence criterion is the non-vanishing of a Jacobian determinant, which is hard to decide when the involved polynomials are not given explicitly. Using the double-argument formulae (3.1) for the theta-constants we construct suitable polynomials $P_{2^{m}}(X, Y)$ 
(Lemma 3.1). In this case the polynomials $P_{2^{m}}(X, Y)$ are given recursively such that we can solve the problem of the algebraic independence of $\theta(q)$ and $\theta\left(q^{2^{m}}\right)$ for arbitrary integers $m \geqslant 1$. But this method cannot be extended to decide on the algebraic independence of $\theta(q)$ and $\theta\left(q^{n}\right)$ for arbitrary odd integers $n$ by Theorem A. So the main results of this paper are given by the following theorem.

Theorem 1.1. Let $q$ be an algebraic number with $0<|q|<1$. Let $m \geqslant 1$ be an integer. Then, the two numbers $\theta(q)$ and $\theta\left(q^{2^{m}}\right)$ are algebraically independent over $\mathbb{Q}$ as well as the two numbers $\theta(q)$ and $\theta\left(q^{n}\right)$ for $n=3,5,6,7,9,10,11,12$.

Let $n \geqslant 3$ be any odd integer. If the polynomial $P_{n}(X, Y)$ from Theorem $\mathrm{A}$ is given explicitly, then by Theorem 4.1 in Section 4 one can decide on the algebraic independence of $\theta(q)$ and $\theta\left(q^{n}\right)$ over $\mathbb{Q}$ for any algebraic number $q$ satisfying the condition of Theorem 1.1.

The following identities are originally due to Ramanujan (cf. [1, §19, Entries 8 and 17]):

$$
\begin{aligned}
1+S_{1}(q) & =1+\sum_{j=1}^{\infty}(-1)^{j}\left(\frac{j}{5}\right) \frac{j q^{j}}{1-q^{j}}=\frac{1}{4}\left(5 \theta(-q) \theta^{3}\left(-q^{5}\right)-\theta^{3}(-q) \theta\left(-q^{5}\right)\right), \\
24+40 S_{2}(q) & =24+40 \sum_{\substack{j=1 \\
j \equiv 1(2)}}^{\infty}\left(\frac{j}{5}\right) \frac{j q^{j}}{1+q^{j}}=25 \theta(q) \theta^{3}\left(q^{5}\right)-\frac{\theta^{5}(q)}{\theta\left(q^{5}\right)}, \\
1+2 S_{3}(q) & =1+2 \sum_{j=1}^{\infty} \varepsilon_{j} \frac{q^{j}}{1-q^{j}}=\theta(q) \theta\left(q^{7}\right),
\end{aligned}
$$

where $(j / 5)$ denotes the Legendre symbol, and the cycle of coefficients $\left(\varepsilon_{0}, \varepsilon_{1}, \ldots, \varepsilon_{27}\right)$ of length 28 is given by

$$
\begin{aligned}
& (0,1,-1,-1,1,-1,1,0,1,1,1,1,-1,-1,0) \\
& 1,1,-1,-1,-1,-1,0,-1,1,-1,1,1,-1) .
\end{aligned}
$$

Corollary 1.1. Let $q$ be an algebraic number with $0<|q|<1$. Then the numbers $S_{1}(q), S_{2}(q)$, and $S_{3}(q)$ are transcendental.

From Entry 3 and Entry 4 in $[1, \S 19]$ analogous results can be obtained for modular equations of degree 3 .

\section{Auxiliary results}

A detailed discussion of theta-functions and theta-constants can be found in [4, part2, ch.2] and [9, ch.10]. At first we point out some properties of the functions

$$
X_{0}(\tau) \in\left\{n^{2} \frac{\vartheta_{3}^{4}(n \tau)}{\vartheta_{3}^{4}(\tau)}, \frac{\vartheta_{3}^{2}(n \tau)}{\vartheta_{3}^{2}(\tau)}\right\} \quad \text { and } \quad Y_{0}(\tau) \in\left\{16 \frac{\vartheta_{2}^{4}(\tau)}{\vartheta_{3}^{4}(\tau)}, \frac{\vartheta_{4}(\tau)}{\vartheta_{3}(\tau)}\right\}
$$


From the theory of modular forms it is well known that in the upper half plane $\mathbb{H}=\{z \in \mathbb{C} \mid \Im(z)>0\}$ the theta-constants $\vartheta_{2}(\tau), \vartheta_{3}(\tau)$ and $\vartheta_{4}(\tau)$ are regular functions for $\tau \in \mathbb{H}$. Moreover, $\vartheta_{3}(\tau)$ does not vanish in $\mathbb{H}$. Therefore, $X_{0}(\tau)$ and $Y_{0}(\tau)$ are regular functions in $\mathbb{H}$.

The most important tool to transfer the algebraic independence of a set of $m$ numbers to another set of $m$ numbers, which all satisfy a system of algebraic identities, is given by the following lemma. We call it an algebraic independence criterion (AIC).

Lemma 2.1 ([2, Lemma 3.1]). Let $x_{1}, \ldots, x_{m} \in \mathbb{C}$ be algebraically independent over $\mathbb{Q}$ and let $y_{1}, \ldots, y_{m} \in \mathbb{C}$ satisfy the system of equations

$$
f_{j}\left(x_{1}, \ldots, x_{m}, y_{1}, \ldots, y_{m}\right)=0 \quad(1 \leqslant j \leqslant m)
$$

where $f_{j}\left(t_{1}, \ldots, t_{m}, u_{1}, \ldots, u_{m}\right) \in \mathbb{Q}\left[t_{1}, \ldots, t_{m}, u_{1}, \ldots, u_{m}\right](1 \leqslant j \leqslant m)$. Assume that

$$
\operatorname{det}\left(\frac{\partial f_{j}}{\partial t_{i}}\left(x_{1}, \ldots, x_{m}, y_{1}, \ldots, y_{m}\right)\right) \neq 0
$$

Then the numbers $y_{1}, \ldots, y_{m}$ are algebraically independent over $\mathbb{Q}$.

We shall apply the AIC to the sets $\left\{x_{1}, x_{2}\right\}=\left\{\vartheta_{2}(\tau), \vartheta_{3}(\tau)\right\}$ and $\left\{x_{1}, x_{2}\right\}=$ $\left\{\vartheta_{3}(\tau), \vartheta_{4}(\tau)\right\}$. For this purpose we have to know that these pairs of numbers are algebraically independent.

Lemma 2.2 ([3, Lemma 4]). Let $q$ be an algebraic number with $q=e^{\pi i \tau}$ and $\Im(\tau)>0$. Then, the numbers in each of the sets

$$
\left\{\vartheta_{2}(\tau), \vartheta_{3}(\tau)\right\}, \quad\left\{\vartheta_{2}(\tau), \vartheta_{4}(\tau)\right\}, \quad\left\{\vartheta_{3}(\tau), \vartheta_{4}(\tau)\right\}
$$

are algebraically independent over $\mathbb{Q}$.

This result can be derived from Yu.V.Nesterenko's theorem [7] on the algebraic independence of the values $P(q), Q(q), R(q)$ of the Ramanujan functions $P, Q, R$ at a nonvanishing algebraic point $q$.

\section{Preparation of the proof of Theorem 1.1}

In this section, we prove the following lemmas which are required to prove Theorem 1.1 when $n$ is a power of two.

Lemma 3.1. For every integer $m \geqslant 1$ let $n=2^{m}$. There exists a polynomial $P_{n}(X, Y) \in \mathbb{Z}[X, Y]$ such that

$$
P_{n}\left(\frac{\vartheta_{3}^{2}(n \tau)}{\vartheta_{3}^{2}(\tau)}, \frac{\vartheta_{4}(\tau)}{\vartheta_{3}(\tau)}\right)=0
$$

with $\operatorname{deg}_{X} P_{2}(X, Y)=1$, and $\operatorname{deg}_{X} P_{n}(X, Y)=2^{m-2}$ for $m \geqslant 2$. 
Proof. For simplicity we introduce the notation $\vartheta_{3}:=\vartheta_{3}(\tau)$ and $\vartheta_{4}:=\vartheta_{4}(\tau)$. Then

$$
\left.\begin{array}{rl}
2 \vartheta_{2}^{2}(2 \tau) & =\vartheta_{3}^{2}-\vartheta_{4}^{2} \\
2 \vartheta_{3}^{2}(2 \tau) & =\vartheta_{3}^{2}+\vartheta_{4}^{2} \\
\vartheta_{4}^{2}(2 \tau) & =\vartheta_{3} \vartheta_{4}
\end{array}\right\}
$$

For every integer $m \geqslant 1$ let

$$
\begin{aligned}
& z_{1}:=\vartheta_{3}^{2}(n \tau), \\
& z_{2}:=\left(\vartheta_{3}+\vartheta_{4}\right)^{2}, \\
& z_{3}:=\vartheta_{3} \vartheta_{4} .
\end{aligned}
$$

Note that $z_{1}$ depends on $n=2^{m}$, while $z_{2}$ and $z_{3}$ do not depend on $n$. First, we compute the polynomials $P_{n}(X, Y)$ for $n=2,4,8$.

$n=2$ : From (3.1) we have

$$
2 \vartheta_{3}^{2}(2 \tau)-\left(\vartheta_{3}+\vartheta_{4}\right)^{2}+2 \vartheta_{3} \vartheta_{4}=2 z_{1}-z_{2}+2 z_{3}=0
$$

Dividing by $\vartheta_{3}^{2}$, it follows that

$$
2\left(\frac{\vartheta_{3}(2 \tau)}{\vartheta_{3}}\right)^{2}-\left(1+\frac{\vartheta_{4}}{\vartheta_{3}}\right)^{2}+2 \frac{\vartheta_{4}}{\vartheta_{3}}=0 .
$$

Hence, $P_{2}(X, Y)=2 X-(1+Y)^{2}+2 Y$.

$n=4:$ In the second identity of (3.1) we replace $\tau$ by $2 \tau$ and then express $\vartheta_{3}^{2}(2 \tau)$ and $\vartheta_{4}^{2}(2 \tau)$ on the right-hand side again by (3.1) in terms of $\vartheta_{3}$ and $\vartheta_{4}$ :

$$
4 \vartheta_{3}^{2}(4 \tau)-\left(\vartheta_{3}+\vartheta_{4}\right)^{2}=4 z_{1}-z_{2}=0
$$

Dividing by $\vartheta_{3}^{2}$, it follows that

$$
4\left(\frac{\vartheta_{3}(4 \tau)}{\vartheta_{3}}\right)^{2}-\left(1+\frac{\vartheta_{4}}{\vartheta_{3}}\right)^{2}=0 .
$$

Hence, $P_{4}(X, Y)=4 X-(1+Y)^{2}$.

$n=8$ : In $(3.3)$ we replace $\tau$ by $2 \tau$. In order to express $\vartheta_{3}(2 \tau)$ and $\vartheta_{4}(2 \tau)$ in terms of $\vartheta_{3}$ and $\vartheta_{4}$, it becomes necessary to solve the identity for $\vartheta_{3} \vartheta_{4}$ and square the equation. After some straightforward computations it turns out that

$$
\begin{aligned}
0 & =\left(8 \vartheta_{3}^{2}(8 \tau)-\left(\vartheta_{3}+\vartheta_{4}\right)^{2}\right)^{2}-8\left(\left(\vartheta_{3}+\vartheta_{4}\right)^{2}-2 \vartheta_{3} \vartheta_{4}\right) \vartheta_{3} \vartheta_{4} \\
& =\left(8 z_{1}-z_{2}\right)^{2}-8\left(z_{2}-2 z_{3}\right) z_{3} .
\end{aligned}
$$

Dividing by $\vartheta_{3}^{4}$, we find that

$$
P_{8}(X, Y)=\left(8 X-(1+Y)^{2}\right)^{2}-8\left((1+Y)^{2}-2 Y\right) Y
$$


The polynomials in terms of $z_{1}, z_{2}, z_{3}$ in $(3.2-3.4)$ are homogeneous of degrees 1,1 , and 2 respectively. Therefore, we try to prove the following statement by induction with respect to $m$ :

For every $m \geqslant 1$ there is a homogeneous polynomial $T_{n}\left(t_{1}, t_{2}, t_{3}\right) \in \mathbb{Z}\left[t_{1}, t_{2}, t_{3}\right]$ of total degree $\lambda$ such that $T_{n}\left(z_{1}, z_{2}, z_{3}\right)=0$ with $\lambda=\operatorname{deg}_{t_{1}} T_{n}\left(t_{1}, t_{2}, t_{3}\right)=2^{m-2}$ for $m \geqslant 2$ and $\lambda=1$ when $m=1$.

We have already shown the existence of $T_{2}, T_{4}$, and $T_{8}$. For $T_{8}$ we have $\lambda=2$ by (3.4). So, let us assume that for some $m \geqslant 3$ such a homogeneous polynomial $T_{2^{m}}$ with $\lambda=2^{m-2}$ do exist. Then,

$$
T_{2^{m}}\left(\vartheta_{3}^{2}\left(2^{m} \tau\right),\left(\vartheta_{3}+\vartheta_{4}\right)^{2}, \vartheta_{3} \vartheta_{4}\right)=0
$$

where

$$
T_{2^{m}}\left(t_{1}, t_{2}, t_{3}\right)=\sum_{\nu} a_{\nu} t_{1}^{\nu_{1}} t_{2}^{\nu_{2}} t_{3}^{\nu_{3}}
$$

say, with $\nu=\left(\nu_{1}, \nu_{2}, \nu_{3}\right) \in \mathbb{N}^{3}, a_{\nu} \in \mathbb{Z}$, and $\nu_{1}+\nu_{2}+\nu_{3}=\lambda=\operatorname{deg}_{t_{1}} T_{2^{m}}\left(t_{1}, t_{2}, t_{3}\right)$. Here, $\mathbb{N}$ denotes the set of nonnegative integers. The leading term with respect to $t_{1}$ occurs once only for $\nu=(\lambda, 0,0)$. Next, in (3.5) we replace $\tau$ by $2 \tau$ :

$$
T_{2^{m}}\left(\vartheta_{3}^{2}\left(2^{m+1} \tau\right),\left(\vartheta_{3}(2 \tau)+\vartheta_{4}(2 \tau)\right)^{2}, \vartheta_{3}(2 \tau) \vartheta_{4}(2 \tau)\right)=0
$$

Setting $w:=\vartheta_{3}(2 \tau) \vartheta_{4}(2 \tau)$, we have, using (3.1),

$$
\left(\vartheta_{3}(2 \tau)+\vartheta_{4}(2 \tau)\right)^{2}=\frac{z_{2}}{2}+2 w
$$

For $m+1$ we set $z_{1}:=\vartheta_{3}^{2}\left(2^{m+1} \tau\right)=\vartheta_{3}^{2}((n+1) \tau)$. Then, (3.7) and (3.6) can be expressed in terms of $z_{1}, z_{2}$, and $w$ :

$$
\begin{aligned}
0 & =T_{2^{m}}\left(z_{1}, \frac{z_{2}}{2}+2 w, w\right) \\
& =\sum_{\nu} a_{\nu} z_{1}^{\nu_{1}}\left(\frac{z_{2}}{2}+2 w\right)^{\nu_{2}} w^{\nu_{3}} \\
& =\sum_{\mu} b_{\mu} z_{1}^{\mu_{1}} z_{2}^{\mu_{2}} w^{\mu_{3}}
\end{aligned}
$$

with $\mu=\left(\mu_{1}, \mu_{2}, \mu_{3}\right) \in \mathbb{N}^{3}, b_{\mu} \in \mathbb{Q}$, and $\mu_{1}+\mu_{2}+\mu_{3}=\lambda$. We separate the sum on $\mu=\left(\mu_{1}, \mu_{2}, \mu_{3}\right)$ into two parts according to the parity of $\mu_{3}$ :

$$
0=\sum_{\substack{\mu=\left(\mu_{1}, \mu_{2}, \mu_{3}\right) \\ \mu_{3} \equiv 0(\bmod 2)}} b_{\mu} z_{1}^{\mu_{1}} z_{2}^{\mu_{2}} w^{\mu_{3}}+\sum_{\substack{\mu=\left(\mu_{1}, \mu_{2}, \mu_{3}\right) \\ \mu_{3} \equiv 1(\bmod 2)}} b_{\mu} z_{1}^{\mu_{1}} z_{2}^{\mu_{2}} w^{\mu_{3}},
$$


where the leading term with respect to $z_{1}$ is $b_{(\lambda, 0,0)} z_{1}^{\lambda} \not \equiv 0$ occurring in the lefthand sum. It follows that

$$
\left(\sum_{\substack{\mu=\left(\mu_{1}, \mu_{2}, \mu_{3}\right) \\ \mu_{3} \equiv 0(\bmod 2)}} b_{\mu} z_{1}^{\mu_{1}} z_{2}^{\mu_{2}} w^{\mu_{3}}\right)^{2}-w^{2}\left(\sum_{\substack{\mu_{0}=\left(\mu_{1}, \mu_{2}, \mu_{3}\right) \\ \mu_{3} \equiv 1(\bmod 2)}} b_{\mu} z_{1}^{\mu_{1}} z_{2}^{\mu_{2}} w^{\mu_{3}-1}\right)^{2}=0 .
$$

Using (3.1) we express $w^{2}$ in terms of $z_{2}$ and $z_{3}$ :

$$
w^{2}=\frac{1}{2}\left(z_{2}-2 z_{3}\right) z_{3}
$$

Substituting this expression into (3.8), we obtain

$$
\begin{aligned}
0= & \left(\sum_{\substack{\mu=\left(\mu_{1}, \mu_{2}, \mu_{3}\right) \\
\mu_{3} \equiv 0(\bmod 2)}} b_{\mu} z_{1}^{\mu_{1}} z_{2}^{\mu_{2}} 2^{-\mu_{3} / 2}\left(z_{2}-2 z_{3}\right)^{\mu_{3} / 2} z_{3}^{\mu_{3} / 2}\right)^{2} \\
& -\frac{1}{2}\left(z_{2}-2 z_{3}\right) z_{3}\left(\sum_{\substack{\mu=\left(\mu_{1}, \mu_{2}, \mu_{3}\right) \\
\mu_{3} \equiv 1(\bmod 2)}} b_{\mu} z_{1}^{\mu_{1}} z_{2}^{\mu_{2}} 2^{-\left(\mu_{3}-1\right) / 2}\left(z_{2}-2 z_{3}\right)^{\left(\mu_{3}-1\right) / 2} z_{3}^{\left(\mu_{3}-1\right) / 2}\right)^{2} \\
= & \sum_{\kappa} c_{\kappa} z_{1}^{\kappa_{1}} z_{2}^{\kappa_{2}} z_{3}^{\kappa_{3}},
\end{aligned}
$$

where $\kappa=\left(\kappa_{1}, \kappa_{2}, \kappa_{3}\right) \in \mathbb{N}^{3}, c_{\kappa} \in \mathbb{Q}$, and $\kappa_{1}+\kappa_{2}+\kappa_{3}=2 \lambda$. The leading term with respect to $z_{1}$ is $c_{(2 \lambda, 0,0)} z_{1}^{2 \lambda} \not \equiv 0$. The homogeneous polynomial $T_{2^{m+1}} \in$ $\mathbb{Z}\left[t_{1}, t_{2}, t_{3}\right] \backslash\{0\}$ can be chosen by

$$
T_{2^{m+1}}\left(t_{1}, t_{2}, t_{3}\right):=2^{2 \lambda} \sum_{\kappa} c_{\kappa} t_{1}^{\kappa_{1}} t_{2}^{\kappa_{2}} t_{3}^{\kappa_{3}} .
$$

For this polynomial we have $2 \lambda=2^{m-1}$. This completes the proof of the existence of the homogeneous polynomials $T_{n}\left(t_{1}, t_{2}, t_{3}\right)$ for every integer $m \geqslant 1$ with $n=2^{m}$ satisfying $T_{n}\left(z_{1}, z_{2}, z_{3}\right)=0$. Let us consider a monomial of such a homogeneous polynomial $T_{n}$ of degree $\lambda$ given by (3.6). Then we have $\nu_{1}+\nu_{2}+\nu_{3}=\lambda$. After dividing $T_{n}$ by $\vartheta_{3}^{2 \lambda}$, the monomial takes the form

$$
\begin{aligned}
\frac{a_{\nu}}{\vartheta_{3}^{2 \lambda}} \cdot z_{1}^{\nu_{1}} z_{2}^{\nu_{2}} z_{3}^{\nu_{3}} & =\frac{a_{\nu}}{\vartheta_{3}^{2 \lambda}} \cdot\left(\vartheta_{3}\left(2^{m} \tau\right)\right)^{2 \nu_{1}}\left(\vartheta_{3}+\vartheta_{4}\right)^{2 \nu_{2}}\left(\vartheta_{3} \vartheta_{4}\right)^{\nu_{3}} \\
& =a_{\nu}\left(\frac{\vartheta_{3}\left(2^{m} \tau\right)}{\vartheta_{3}}\right)^{2 \nu_{1}}\left(1+\frac{\vartheta_{4}}{\vartheta_{3}}\right)^{2 \nu_{2}}\left(\frac{\vartheta_{4}}{\vartheta_{3}}\right)^{\nu_{3}} \\
& =a_{\nu} X^{\nu_{1}}(1+Y)^{2 \nu_{2}} Y^{\nu_{3}}
\end{aligned}
$$

with

$$
X:=\frac{\vartheta_{3}^{2}\left(2^{m} \tau\right)}{\vartheta_{3}^{2}} \quad \text { and } \quad Y:=\frac{\vartheta_{4}}{\vartheta_{3}} .
$$

Introducing the polynomial

$$
P_{n}(X, Y):=\sum_{\nu} a_{\nu} X^{\nu_{1}}(1+Y)^{2 \nu_{2}} Y^{\nu_{3}}=T_{n}\left(X,(1+Y)^{2}, Y\right),
$$

we finish the proof of Lemma 3.1. 
The polynomials $P_{2}, P_{4}, P_{8}, P_{16}$, and $P_{32}$ are listed in the appendix. The proof of the algebraic independence of $\vartheta_{3}\left(q^{2^{m}}\right)$ and $\vartheta_{3}(q)$ over $\mathbb{Q}$ will require some more information on the polynomials $T_{n}\left(t_{1}, t_{2}, t_{3}\right)$ introduced in the proof of the preceding lemma.

Lemma 3.2. For every integer $m \geqslant 3$ let $n=2^{m}$. Then there is a polynomial $U_{n}\left(t_{1}, t_{2}, t_{3}\right) \in \mathbb{Q}\left[t_{1}, t_{2}, t_{3}\right]$ such that the polynomial $T_{n}\left(t_{1}, t_{2}, t_{3}\right)$ from (3.5) can be written as

$$
T_{n}\left(t_{1}, t_{2}, t_{3}\right)=\left(n t_{1}-t_{2}\right)^{2^{m-2}}+t_{3} U_{n}\left(t_{1}, t_{2}, t_{3}\right)
$$

with

$$
U_{n}\left(\frac{1}{n}, 1,0\right)=-2^{2^{m-1}-1}
$$

Proof. Lemma 3.2 is true for $m=3$ and $m=4$. We have

$$
\begin{aligned}
T_{8}\left(t_{1}, t_{2}, t_{3}\right)= & \left(8 t_{1}-t_{2}\right)^{2}-8\left(t_{2}-2 t_{3}\right) t_{3} \\
U_{8}\left(t_{1}, t_{2}, t_{3}\right)= & -8\left(t_{2}-2 t_{3}\right) \\
T_{16}\left(t_{1}, t_{2}, t_{3}\right)= & \left(16 t_{1}-t_{2}\right)^{4}+t_{3}\left(16\left(t_{2}-2 t_{3}\right)\left(16 t_{1}-t_{2}\right)^{2}+64\left(t_{2}-2 t_{3}\right)^{2} t_{3}\right. \\
& \left.-128\left(t_{2}-2 t_{3}\right)\left(8 t_{1}+\frac{t_{2}}{2}\right)^{2}\right) \\
U_{16}\left(t_{1}, t_{2}, t_{3}\right)= & 16\left(t_{2}-2 t_{3}\right)\left(16 t_{1}-t_{2}\right)^{2}+64\left(t_{2}-2 t_{3}\right)^{2} t_{3} \\
& -128\left(t_{2}-2 t_{3}\right)\left(8 t_{1}+\frac{t_{2}}{2}\right)^{2} .
\end{aligned}
$$

For $m \geqslant 4$ we prove a more precise result on the particular shape of the polynomials $T_{2^{m}}$. We shall show the following. For every integer $m \geqslant 4$ we have

$$
\begin{aligned}
T_{2^{m}}\left(t_{1}, t_{2}, t_{3}\right)= & \left(2^{m} t_{1}-t_{2}\right)^{2^{m-2}} \\
& +t_{3} \sum_{\substack{\nu_{1}, \ldots, \nu_{5} \\
\nu_{1} \geqslant 2 \vee \nu_{5} \geqslant 1 \\
\nu_{2} \geqslant 1}} a_{\nu}\left(2^{m} t_{1}-t_{2}\right)^{\nu_{1}}\left(t_{2}-2 t_{3}\right)^{\nu_{2}} t_{1}^{\nu_{3}} t_{2}^{\nu_{4}} t_{3}^{\nu_{5}} \\
& -2^{2^{m-1}-1} t_{2}^{2^{m-3}-2}\left(t_{2}-2 t_{3}\right)\left(2^{m-1} t_{1}+\frac{t_{2}}{2}\right)^{2^{m-3}} t_{3} .
\end{aligned}
$$

Here, $\nu=\left(\nu_{1}, \ldots, \nu_{5}\right) \in \mathbb{N}^{5}$, and the numbers $a_{\nu}$ are rationals. Only finitely many $a_{\nu}$ do not vanish. One can show that $T_{2^{m}}\left(t_{1}, t_{2}, t_{3}\right)$ is a polynomial with integer coefficients, but we do not need this fact. We point out that the conditions on the summation variables $\nu_{1}, \ldots, \nu_{5}$ read as follows: it is either $\nu_{1} \geqslant 2$ or $\nu_{5} \geqslant 1$ (or both), and we always have $\nu_{2} \geqslant 1$. The second and third term on the righthand side of (3.12) form $t_{3} U_{2^{m}}\left(t_{1}, t_{2}, t_{3}\right)$, which implies (3.9). In particular, for $t_{1}=1 / 2^{m}, t_{2}=1$, and $t_{3}=0$, we have

$$
2^{m} t_{1}-t_{2}=0, \quad t_{2}-2 t_{3}=1, \quad 2^{m-1} t_{1}+\frac{t_{2}}{2}=1,
$$


such that

$$
U_{2^{m}}\left(\frac{1}{2^{m}}, 1,0\right)=-2^{2^{m-1}-1}
$$

proves (3.10) in Lemma 3.2.

Proof of (3.12). We proceed by induction on $m$. For $m=4$ see (3.11). Next let us assume that (3.12) holds for some integer $m \geqslant 4$. Following the lines in the proof of Lemma 3.1, we construct step by step the new polynomial $T_{2^{m+1}}\left(t_{1}, t_{2}, t_{3}\right)$ from (3.12).

Step 1: After substituting the new expressions

$$
t_{1} \rightarrow t_{1}, \quad t_{2} \rightarrow \frac{t_{2}}{2}+2 w, \quad t_{3} \rightarrow w
$$

into (3.12), we see that the resulting term equals to zero. Hence,

$$
\begin{aligned}
0= & \left(2^{m} t_{1}-\frac{t_{2}}{2}-2 w\right)^{2^{m-2}} \\
& +w \sum_{\substack{\nu_{1}, \ldots, \nu_{5} \\
\nu_{1} \geqslant 2 \vee \nu_{5} \geqslant 1 \\
\nu_{2} \geqslant 1}} a_{\nu}\left(2^{m} t_{1}-\frac{t_{2}}{2}-2 w\right)^{\nu_{1}}\left(\frac{t_{2}}{2}\right)^{\nu_{2}} t_{1}^{\nu_{3}}\left(\frac{t_{2}}{2}+2 w\right)^{\nu_{4}} w^{\nu_{5}} \\
& -2^{2^{m-1}-1}\left(\frac{t_{2}}{2}+2 w\right)^{2^{m-3}-2} \frac{t_{2}}{2}\left(2^{m-1} t_{1}+\frac{t_{2}}{4}+w\right)^{2^{m-3}} w .
\end{aligned}
$$

Using four times the binomial theorem, the above expression becomes

$$
\begin{aligned}
& 0=\left(2^{m} t_{1}-\frac{t_{2}}{2}\right)^{2^{m-2}}+\sum_{\mu_{1}=1}^{2^{m-2}}\left(\begin{array}{c}
2^{m-2} \\
\mu_{1}
\end{array}\right)(-1)^{\mu_{1}}\left(2^{m} t_{1}-\frac{t_{2}}{2}\right)^{2^{m-2}-\mu_{1}}(2 w)^{\mu_{1}} \\
& +w \sum_{\substack{\nu_{1}, \ldots, \nu_{5} \\
\nu_{1} \geqslant 2 \vee \nu_{5} \geqslant 1 \\
\nu_{2} \geqslant 1}} a_{\nu}\left(\sum_{\mu_{2}=0}^{\nu_{1}}\left(\begin{array}{c}
\nu_{1} \\
\mu_{2}
\end{array}\right)(-1)^{\mu_{2}}\left(2^{m} t_{1}-\frac{t_{2}}{2}\right)^{\nu_{1}-\mu_{2}}(2 w)^{\mu_{2}}\right)\left(\frac{t_{2}}{2}\right)^{\nu_{2}} t_{1}^{\nu_{3}} \\
& \times\left(\sum_{\mu_{3}=0}^{\nu_{4}}\left(\begin{array}{l}
\nu_{4} \\
\mu_{3}
\end{array}\right)\left(\frac{t_{2}}{2}\right)^{\nu_{4}-\mu_{3}}(2 w)^{\mu_{3}}\right) w^{\nu_{5}} \\
& -2^{2^{m-1}-1}\left(\frac{t_{2}}{2}\right)^{2^{m-3}-2} \frac{t_{2}}{2}\left(2^{m-1} t_{1}+\frac{t_{2}}{4}+w\right)^{2^{m-3}} w \\
& -2^{2^{m-1}-1}\left(\sum_{\mu_{4}=1}^{2^{m-3}-2}\left(\begin{array}{c}
2^{m-3}-2 \\
\mu_{4}
\end{array}\right)\left(\frac{t_{2}}{2}\right)^{2^{m-3}-2-\mu_{4}}(2 w)^{\mu_{4}}\right) \frac{t_{2}}{2} \\
& \times\left(2^{m-1} t_{1}+\frac{t_{2}}{4}+w\right)^{2^{m-3}} w \text {. }
\end{aligned}
$$


The last but one term on the right-hand side of (3.13) can be expanded by

$$
\begin{aligned}
& 2^{2^{m-1}-1}\left(\frac{t_{2}}{2}\right)^{2^{m-3}-2} \frac{t_{2}}{2} \cdot \frac{1}{2^{2^{m-3}}}\left(2^{m} t_{1}+\frac{t_{2}}{2}+2 w\right)^{2^{m-3}} w \\
= & 2^{2^{m-1}-2^{m-3}-2^{m-3}-1-1+2} t_{2}^{2^{m-3}-1}\left(2^{m} t_{1}+\frac{t_{2}}{2}+2 w\right)^{2^{m-3}} w \\
= & 2^{2^{m-2}} t_{2}^{2^{m-3}-1}\left(2^{m} t_{1}+\frac{t_{2}}{2}\right)^{2^{m-3}} w \\
& +2^{2^{m-2}} t_{2}^{2^{m-3}-1}\left(\sum_{\mu_{5}=1}^{2^{m-3}}\left(\begin{array}{c}
2^{m-3} \\
\mu_{5}
\end{array}\right)\left(2^{m} t_{1}+\frac{t_{2}}{2}\right)^{2^{m-3}-\mu_{5}}(2 w)^{\mu_{5}}\right) w .
\end{aligned}
$$

Substituting (3.14) for the last but one term into (3.13), we summarize the terms as follows.

$$
\begin{aligned}
0= & \left(2^{m} t_{1}-\frac{t_{2}}{2}\right)^{2^{m-2}}+\sum_{\substack{\mu_{6}, \ldots, \mu_{9} \\
\mu_{6} \geqslant 2 \vee \mu_{9} \geqslant 2 \\
\mu_{9} \geqslant 1}} b_{\mu}\left(2^{m+1} t_{1}-t_{2}\right)^{\mu_{6}} t_{1}^{\mu_{7}} t_{2}^{\mu_{8}} w^{\mu_{9}} \\
& +2^{2^{m-2}} t_{2}^{2^{m-3}-1}\left(2^{m} t_{1}+\frac{t_{2}}{2}\right)^{2^{m-3}} w
\end{aligned}
$$

Here, we abbreviate by $\mu=\left(\mu_{6}, \ldots, \mu_{9}\right)$, and the coefficients $b_{\mu}$ are again rational numbers.

Step 2: In (3.15) we separate the terms with an even power of $w$ from those with an odd power of $w$. This gives

$$
\begin{aligned}
& \left(2^{m} t_{1}-\frac{t_{2}}{2}\right)^{2^{m-2}}+\sum_{\substack{\mu_{6}, \ldots, \mu_{9} \\
\mu_{9} \geqslant 2 \\
(\bmod 2)}} b_{\mu}\left(2^{m+1} t_{1}-t_{2}\right)^{\mu_{6}} t_{1}^{\mu_{7}} t_{2}^{\mu_{8}} w^{\mu_{9}} \\
= & \sum_{\substack{\mu_{6}, \ldots, \mu_{9} \\
\mu_{6} \geqslant 2 \vee \mu_{9} \geqslant 2 \\
\mu_{9} \equiv 1(\bmod 2) \\
\mu_{9} \geqslant 1}} b_{\mu}\left(2^{m+1} t_{1}-t_{2}\right)^{\mu_{6}} t_{1}^{\mu_{7}} t_{2}^{\mu_{8}} w^{\mu_{9}} \\
- & 2^{2^{m-2}} t_{2}^{2^{m-3}-1}\left(2^{m} t_{1}+\frac{t_{2}}{2}\right)^{2^{m-3}} w .
\end{aligned}
$$


Step 3: Squaring (3.16), we obtain

$$
\begin{aligned}
& \left(2^{m} t_{1}-\frac{t_{2}}{2}\right)^{2^{m-1}}+\left(\sum_{\substack{\mu_{6}, \ldots, \mu_{9} \\
\mu_{9} \geqslant 2}} b_{\mu}\left(2^{m+1} t_{1}-t_{2}\right)^{\mu_{6}} t_{1}^{\mu_{7}} t_{2}^{\mu_{8}} w^{\mu_{9}}\right)^{2} \\
& +2\left(2^{m} t_{1}-\frac{t_{2}}{2}\right)^{2^{m-2}} \sum_{\substack{\mu_{6}, \ldots, \mu_{9} \\
\mu_{9} \geqslant 2 \\
\mu_{9} \equiv 0(\bmod 2)}} b_{\mu}\left(2^{m+1} t_{1}-t_{2}\right)^{\mu_{6}} t_{1}^{\mu_{7}} t_{2}^{\mu_{8}} w^{\mu_{9}} \\
& =\left(\sum_{\substack{\mu_{6}, \ldots, \mu_{9} \\
\mu_{6} \geqslant 2 \vee \mu_{9} \geqslant 2 \\
\mu_{9} \equiv 1(\bmod 2) \\
\mu_{9} \geqslant 1}} b_{\mu}\left(2^{m+1} t_{1}-t_{2}\right)^{\mu_{6}} t_{1}^{\mu_{7}} t_{2}^{\mu_{8}} w^{\mu_{9}}\right)^{2} \\
& +2^{2^{m-2}+1} t_{2}^{2^{m-3}-1}\left(2^{m} t_{1}+\frac{t_{2}}{2}\right)^{2^{m-3}} \sum_{\substack{\mu_{6}, \ldots, \mu_{9} \\
\mu_{6} \geqslant 2 \vee \mu_{9} \geqslant 2 \\
\mu_{9} \equiv 1(\bmod 2) \\
\mu_{9} \geqslant 1}} b_{\mu}\left(2^{m+1} t_{1}-t_{2}\right)^{\mu_{6}} t_{1}^{\mu_{7}} t_{2}^{\mu_{8}} w^{1+\mu_{9}} \\
& +2^{2^{m-1}} t_{2}^{2^{m-2}-2}\left(2^{m} t_{1}+\frac{t_{2}}{2}\right)^{2^{m-2}} w^{2} .
\end{aligned}
$$

This identity can be summarized as follows.

$$
\begin{aligned}
0= & \left(2^{m} t_{1}-\frac{t_{2}}{2}\right)^{2^{m-1}}+\sum_{\substack{\nu_{6}, \ldots, \nu_{9} \\
\nu_{6} \geqslant 2 \vee \nu_{7} \geqslant 2 \\
\nu_{7} \geqslant 1}} c_{\nu}\left(2^{m} t_{1}-\frac{t_{2}}{2}\right)^{\nu_{6}} w^{2 \nu_{7}} t_{1}^{\nu_{8}} t_{2}^{\nu_{9}} \\
& -2^{2^{m-1}} t_{2}^{2^{m-2}-2}\left(2^{m} t_{1}+\frac{t_{2}}{2}\right)^{2^{m-2}} w^{2},
\end{aligned}
$$

where $\nu=\left(\nu_{6}, \ldots, \nu_{9}\right)$ and $c_{\nu} \in \mathbb{Q}$.

Step 4: Multiplying (3.17) by $2^{2^{m-1}}$ and replacing $w^{2}$ by $\frac{1}{2}\left(t_{2}-2 t_{3}\right) t_{3}$, the right-hand side of $(3.17)$ becomes the polynomial $T_{2^{m+1}}\left(t_{1}, t_{2}, t_{3}\right)$. Thus we obtain 


$$
\begin{aligned}
T_{2^{m+1}}\left(t_{1}, t_{2}, t_{3}\right)= & \left(2^{m+1} t_{1}-t_{2}\right)^{2^{m-1}} \\
& +\sum_{\substack{\nu_{6}, \ldots, \nu_{9} \\
\nu_{6} \geqslant 2 \vee \nu_{7} \geqslant 2 \\
\nu_{7} \geqslant 1}} 2^{2^{m-1}} c_{\nu}\left(2^{m} t_{1}-\frac{t_{2}}{2}\right)^{\nu_{6}} \frac{1}{2^{\nu_{7}}}\left(t_{2}-2 t_{3}\right)^{\nu_{7}} t_{3}^{\nu_{7}} t_{1}^{\nu_{8}} t_{2}^{\nu_{9}} \\
& -2^{2^{m-1}+2^{m-1}} t_{2}^{2^{m-2}-2}\left(2^{m} t_{1}+\frac{t_{2}}{2}\right)^{2^{m-2}} \frac{1}{2}\left(t_{2}-2 t_{3}\right) t_{3} \\
= & \left(2^{m+1} t_{1}-t_{2}\right)^{2^{m-1}} \\
& +t_{3} \sum_{\substack{\nu_{6} \geqslant 2 \vee, \nu_{10} \\
\nu_{7} \geqslant 1}} d_{\nu}\left(2^{m+1} t_{1}-t_{2}\right)^{\nu_{6}}\left(t_{2}-2 t_{3}\right)^{\nu_{7}} t_{1}^{\nu_{8}} t_{2}^{\nu_{9}} t_{3}^{\nu_{10}} \\
& -2^{2^{m}-1} t_{2}^{2^{m-2}-2}\left(t_{2}-2 t_{3}\right)\left(2^{m} t_{1}+\frac{t_{2}}{2}\right)^{2^{m-2}} t_{3}
\end{aligned}
$$

where $\nu=\left(\nu_{6}, \ldots, \nu_{10}\right)$, and $d_{\nu} \in \mathbb{Q}$. Hence, (3.18) corresponds to (3.12) with $m$ replaced by $m+1$. This proves $(3.12)$.

\section{Proof of Theorem 1.1}

By $\operatorname{Res}_{t}(f(t), g(t))$ we denote the resultant of two polynomials $f(t), g(t)$ with respect to the variable $t$. It is consistent with the notation of theta-constants to write $\vartheta_{3}(q)$ and $\vartheta_{3}(\tau)$ instead of $\theta(q)$ and $\theta(\tau)$, respectively.

We divide the proof of Theorem 1.1 into several steps. The first step is an interim result given by the following theorem.

Theorem 4.1. Let $n$ be either an odd integer $\geqslant 3$ or $n=2^{m}$ with $m \geqslant 1$. Let $q$ be an algebraic number with $q=e^{\pi i \tau}$ and $\Im(\tau)>0$. If the polynomial

$$
\operatorname{Res}_{X}\left(P_{n}(X, Y), \frac{\partial}{\partial Y} P_{n}(X, Y)\right)
$$

does not vanish identically, then the numbers $\vartheta_{3}(n \tau)$ and $\vartheta_{3}(\tau)$ are algebraically independent over $\mathbb{Q}$.

Proof. For any given odd integer $n \geqslant 3$ let

$$
\begin{aligned}
X_{0}:=n^{2} \frac{\vartheta_{3}^{4}(n \tau)}{\vartheta_{3}^{4}(\tau)}, & Y_{0}:=16 \frac{\vartheta_{2}^{4}(\tau)}{\vartheta_{3}^{4}(\tau)} ; \\
x_{1}:=\vartheta_{2}(\tau), & x_{2}:=\vartheta_{3}(\tau), \\
y_{1}:=\vartheta_{3}(n \tau), & y_{2}:=x_{2}=\vartheta_{3}(\tau) .
\end{aligned}
$$

We know by Theorem A that $P_{n}\left(X_{0}, Y_{0}\right)=0$, and by Lemma 2.2 and the conditions of Theorem 4.1 that $x_{1}$ and $x_{2}$ are algebraically independent over $\mathbb{Q}$. Let

$$
P_{n}(X, Y)=\sum_{\nu=0}^{N} \sum_{\mu=0}^{M} a_{\nu, \mu} X^{\nu} Y^{\mu}
$$


where $a_{\nu, \mu}$ are the integer coefficients of the polynomial $P_{n}$. Consider the polynomials

$$
\begin{aligned}
f_{1}:=f_{1}\left(t_{1}, t_{2}, u_{1}, u_{2}\right): & =t_{2}^{4 M} u_{2}^{4 N} P_{n}\left(\frac{n^{2} u_{1}^{4}}{u_{2}^{4}}, \frac{16 t_{1}^{4}}{t_{2}^{4}}\right) \\
& =\sum_{\nu=0}^{N} \sum_{\mu=0}^{M} a_{\nu, \mu} t_{2}^{4 M} u_{2}^{4 N}\left(\frac{n^{2} u_{1}^{4}}{u_{2}^{4}}\right)^{\nu}\left(\frac{16 t_{1}^{4}}{t_{2}^{4}}\right)^{\mu} \\
& =\sum_{\nu=0}^{N} \sum_{\mu=0}^{M} 16^{\mu} n^{2 \nu} a_{\nu, \mu} t_{1}^{4 \mu} t_{2}^{4(M-\mu)} u_{1}^{4 \nu} u_{2}^{4(N-\nu)}, \\
f_{2}:=f_{2}\left(t_{1}, t_{2}, u_{1}, u_{2}\right): & =u_{2}-t_{2} .
\end{aligned}
$$

Note that $f_{j}\left(x_{1}, x_{2}, y_{1}, y_{2}\right)=0$ for $j=1,2$. To prove the algebraic independence of $y_{1}$ and $y_{2}$ using the AIC (Lemma 2.1) we have to show that the determinant

$$
\Delta:=\operatorname{det}\left(\begin{array}{ll}
\frac{\partial f_{1}}{\partial t_{1}} & \frac{\partial f_{1}}{\partial t_{2}} \\
\frac{\partial f_{2}}{\partial t_{1}} & \frac{\partial f_{2}}{\partial t_{2}}
\end{array}\right)
$$

does not vanish at $\left(x_{1}, x_{2}, y_{1}, y_{2}\right)$. Since

$$
\frac{\partial f_{2}}{\partial t_{1}}=0 \quad \text { and } \quad \frac{\partial f_{2}}{\partial t_{2}}=-1
$$

the condition $\Delta \neq 0$ is equivalent with the nonvanishing of the number

$$
\frac{\partial f_{1}}{\partial t_{1}}\left(x_{1}, x_{2}, y_{1}, y_{2}\right):=\left.\frac{\partial f_{1}\left(t_{1}, t_{2}, u_{1}, u_{2}\right)}{\partial t_{1}}\right|_{\left(t_{1}=x_{1}, t_{2}=x_{2}, u_{1}=y_{1}, u_{2}=y_{2}\right)} .
$$

We have

$$
\begin{aligned}
\frac{\partial f_{1}}{\partial t_{1}}\left(x_{1}, x_{2}, y_{1}, y_{2}\right) & =\sum_{\nu=0}^{N} \sum_{\mu=1}^{M} 16^{\mu} n^{2 \nu} a_{\nu, \mu} 4 \mu x_{1}^{4 \mu-1} x_{2}^{4(M-\mu)} y_{1}^{4 \nu} y_{2}^{4(N-\nu)} \\
& =x_{2}^{4 M} y_{2}^{4 N} \sum_{\nu=0}^{N} \sum_{\mu=1}^{M} a_{\nu, \mu}\left(\frac{n^{2} y_{1}^{4}}{y_{2}^{4}}\right)^{\nu} \mu\left(\frac{16 x_{1}^{4}}{x_{2}^{4}}\right)^{\mu-1}\left(64 \frac{x_{1}^{3}}{x_{2}^{4}}\right) \\
& =64 x_{1}^{3} x_{2}^{4 M-4} y_{2}^{4 N} \frac{\partial P_{n}}{\partial Y}\left(\frac{n^{2} y_{1}^{4}}{y_{2}^{4}}, \frac{16 x_{1}^{4}}{x_{2}^{4}}\right) .
\end{aligned}
$$

Since both, $x_{1}$ and $x_{2}\left(=y_{2}\right)$ do not vanish, it is clear that

$$
\Delta \neq 0 \Longleftrightarrow \frac{\partial f_{1}}{\partial t_{1}}\left(x_{1}, x_{2}, y_{1}, y_{2}\right) \neq 0 \quad \Longleftrightarrow \quad \frac{\partial P_{n}}{\partial Y}\left(\frac{n^{2} y_{1}^{4}}{y_{2}^{4}}, \frac{16 x_{1}^{4}}{x_{2}^{4}}\right) \neq 0
$$


By the hypothesis of Theorem 4.1 the polynomial

$$
R=\operatorname{Res}_{X}\left(P_{n}(X, Y), \frac{\partial}{\partial Y} P_{n}(X, Y)\right) \in \mathbb{Z}[Y]
$$

does not vanish identically. For $Y=Y_{0}=16 x_{1}^{4} / x_{2}^{4}$ we have $R \in \mathbb{Q}\left(x_{1}, x_{2}\right)$, so that the algebraic independence of $x_{1}, x_{2}$ proves $R \neq 0$. In particular, $P_{n}(X, Y)$ and $\frac{\partial}{\partial Y} P_{n}(X, Y)$ as polynomials in $X$ have no common root for fixed $Y=Y_{0}=$ $16 x_{1}^{4} / x_{2}^{4}$. Since $P_{n}(X, Y)$ vanishes at $\left(X_{0}, Y_{0}\right)=\left(n^{2} y_{1}^{4} / y_{2}^{4}, 16 x_{1}^{4} / x_{2}^{4}\right)$, it follows that

$$
\frac{\partial P_{n}}{\partial Y}\left(\frac{n^{2} y_{1}^{4}}{y_{2}^{4}}, \frac{16 x_{1}^{4}}{x_{2}^{4}}\right) \neq 0 .
$$

Thus, Theorem 4.1 for odd integers $n \geqslant 3$ follows from (4.2) and the AIC (Lemma 2.1).

In the case $n=2^{m}(m \geqslant 1)$ we introduce the quantities

$$
\begin{array}{rlrl}
X_{0} & :=\frac{\vartheta_{3}^{2}(n \tau)}{\vartheta_{3}^{2}(\tau)}, & Y_{0}:=\frac{\vartheta_{4}(\tau)}{\vartheta_{3}(\tau)} \\
x_{1}:=\vartheta_{4}(\tau), & x_{2}:=\vartheta_{3}(\tau), \\
y_{1}:=\vartheta_{3}(n \tau), & y_{2}:=x_{2}=\vartheta_{3}(\tau) .
\end{array}
$$

Here, we have $P_{n}\left(X_{0}, Y_{0}\right)=0$ by Lemma 3.1 , and

$$
\begin{aligned}
f_{1}\left(t_{1}, t_{2}, u_{1}, u_{2}\right) & :=t_{2}^{M} u_{2}^{2 N} P_{n}\left(\frac{u_{1}^{2}}{u_{2}^{2}}, \frac{t_{1}}{t_{2}}\right)=\sum_{\nu=0}^{N} \sum_{\mu=0}^{M} a_{\nu, \mu} t_{1}^{\mu} t_{2}^{M-\mu} u_{1}^{2 \nu} u_{2}^{2(N-\nu)}, \\
\frac{\partial f_{1}}{\partial t_{1}}\left(x_{1}, x_{2}, y_{1}, y_{2}\right) & =x_{2}^{M-1} y_{2}^{2 N} \frac{\partial P_{n}}{\partial Y}\left(\frac{y_{1}^{2}}{y_{2}^{2}}, \frac{x_{1}}{x_{2}}\right), \\
f_{2}\left(t_{1}, t_{2}, u_{1}, u_{2}\right) & :=u_{2}-t_{2} .
\end{aligned}
$$

Then,

$$
\Delta \neq 0 \Longleftrightarrow \frac{\partial P_{n}}{\partial Y}\left(\frac{y_{1}^{2}}{y_{2}^{2}}, \frac{x_{1}}{x_{2}}\right) \neq 0 .
$$

Using similar arguments as above by considering the particular point $\left(X_{0}, Y_{0}\right)=$ $\left(y_{1}^{2} / y_{2}^{2}, x_{1} / x_{2}\right)$, the algebraic independence of $\vartheta_{3}\left(q^{n}\right)$ and $\vartheta_{3}(q)$ for $n=2^{m}$ can be derived from the AIC.

First, using Theorem 4.1 we prove the algebraic independence of $\vartheta_{3}(q)$ and $\vartheta_{3}\left(q^{n}\right)$ for $n=2,3,4,5,7,8,9,11$ by computing the resultant of the polynomials $P_{n}(X, Y)$ and $\partial P_{n}(X, Y) / \partial Y$. We have to show that these resultants do not vanish identically. So, it suffices to compute the values of the resultants at the point $Y=0$ for $n=3,4,5,7,8,11$ and at the point $Y=2$ for $n=2,9$. Note that 
$\operatorname{Res}_{X}\left(P_{n}(X, Y), \frac{\partial P_{n}(X, Y)}{\partial Y}\right)$ vanishes at $Y=0$ for $n=2,9$.

$$
\begin{aligned}
\operatorname{Res}_{X}\left(P_{2}(X, 2), \frac{\partial P_{2}}{\partial Y}(X, 2)\right) & =-2^{2} \\
\operatorname{Res}_{X}\left(P_{3}(X, 0), \frac{\partial P_{3}}{\partial Y}(X, 0)\right) & =2^{16} \cdot 3^{2} \\
\operatorname{Res}_{X}\left(P_{4}(X, 0), \frac{\partial P_{4}}{\partial Y}(X, 0)\right) & =-2 \\
\operatorname{Res}_{X}\left(P_{5}(X, 0), \frac{\partial P_{5}}{\partial Y}(X, 0)\right) & =2^{60} \cdot 3^{10} \cdot 5^{2} \\
\operatorname{Res}_{X}\left(P_{7}(X, 0), \frac{\partial P_{7}}{\partial Y}(X, 0)\right) & =2^{142} \cdot 3^{14} \cdot 7^{2} \\
\operatorname{Res}_{X}\left(P_{8}(X, 0), \frac{\partial P_{8}}{\partial Y}(X, 0)\right) & =2^{12}, \\
\operatorname{Res}_{X}\left(P_{9}(X, 2), \frac{\partial P_{9}}{\partial Y}(X, 2)\right) & =2^{132} \cdot 3^{96} \cdot 7^{2} \cdot 37^{2} \cdot 193^{2} \cdot 5387^{2} \\
& \times 3683832193^{2} \cdot 94686353323^{2} \\
\operatorname{Res}_{X}\left(P_{11}(X, 0), \frac{\partial P_{11}}{\partial Y}(X, 0)\right) & =2^{336} \cdot 3^{22} \cdot 5^{22} \cdot 11^{2} .
\end{aligned}
$$

Next we prove the algebraic independence of the numbers in each of the sets

$$
\left\{\vartheta_{3}(6 \tau), \vartheta_{3}(\tau)\right\} \quad \text { and } \quad\left\{\vartheta_{3}(10 \tau), \vartheta_{3}(\tau)\right\}
$$

We shall not treat these two problems by the method shown before, but again the AIC will play an important role. We first consider the numbers $\vartheta_{3}(6 \tau)$ and $\vartheta_{3}(\tau)$. Given any odd integer $n \geqslant 3$ one can deduce the algebraic independence of $\vartheta_{3}(2 n \tau)$ and $\vartheta_{3}(\tau)$ as follows. First we replace $\tau$ by $2 \tau$ in Theorem A. Then,

$$
P_{n}(X, Y)=0
$$

holds for

$$
X_{0}:=n^{2} \frac{\vartheta_{3}^{4}(2 n \tau)}{\vartheta_{3}^{4}(2 \tau)} \quad \text { and } \quad Y_{0}:=16 \frac{\vartheta_{2}^{4}(2 \tau)}{\vartheta_{3}^{4}(2 \tau)}
$$

Next we express $\vartheta_{2}^{4}(2 \tau)$ and $\vartheta_{3}^{4}(2 \tau)$ in terms of $\vartheta_{3}(\tau)$ and $\vartheta_{4}(\tau)$ :

$$
\begin{aligned}
& \vartheta_{2}^{4}(2 \tau)=\frac{1}{4}\left(\vartheta_{3}^{2}(\tau)-\vartheta_{4}^{2}(\tau)\right)^{2}, \\
& \vartheta_{3}^{4}(2 \tau)=\frac{1}{4}\left(\vartheta_{3}^{2}(\tau)+\vartheta_{4}^{2}(\tau)\right)^{2} .
\end{aligned}
$$

Hence (4.3) holds for

$$
X_{0}=\frac{4 n^{2} \vartheta_{3}^{4}(2 n \tau)}{\left(\vartheta_{3}^{2}(\tau)+\vartheta_{4}^{2}(\tau)\right)^{2}} \quad \text { and } \quad Y_{0}=\frac{16\left(\vartheta_{3}^{2}(\tau)-\vartheta_{4}^{2}(\tau)\right)^{2}}{\left(\vartheta_{3}^{2}(\tau)+\vartheta_{4}^{2}(\tau)\right)^{2}}
$$


Setting

$$
\begin{array}{ll}
x_{1}:=\vartheta_{3}(\tau), & x_{2}:=\vartheta_{4}(\tau), \\
y_{1}:=\vartheta_{3}(2 n \tau), & y_{2}:=x_{1}=\vartheta_{3}(\tau),
\end{array}
$$

we know that (4.3) holds for

$$
X_{0}=\frac{4 n^{2} y_{1}^{4}}{\left(y_{2}^{2}+x_{2}^{2}\right)^{2}} \quad \text { and } \quad Y_{0}=\frac{16\left(x_{1}^{2}-x_{2}^{2}\right)^{2}}{\left(x_{1}^{2}+x_{2}^{2}\right)^{2}}
$$

Beside (4.3) we have the identity $y_{2}-x_{1}=0$, and the numbers $x_{1}, x_{2}$ are known to be algebraically independent over $\mathbb{Q}$ for any algebraic number $q=e^{\pi i \tau}$ with $\Im(\tau)>0$ by Lemma 2.2. Using

$$
P_{n}(X, Y)=\sum_{\nu=1}^{N} \sum_{\mu=1}^{M} a_{\nu, \mu} X^{\nu} Y^{\mu}
$$

we now introduce the polynomials

$$
\begin{aligned}
& f_{1}\left(t_{1}, t_{2}, u_{1}, u_{2}\right):=\left(t_{2}^{2}+u_{2}^{2}\right)^{2 N}\left(t_{1}^{2}+t_{2}^{2}\right)^{2 M} P_{n}\left(\frac{4 n^{2} u_{1}^{4}}{\left(t_{2}^{2}+u_{2}^{2}\right)^{2}}, \frac{16\left(t_{1}^{2}-t_{2}^{2}\right)^{2}}{\left(t_{1}^{2}+t_{2}^{2}\right)^{2}}\right) \\
& f_{2}\left(t_{1}, t_{2}, u_{1}, u_{2}\right):=u_{2}-t_{1}
\end{aligned}
$$

Using the AIC we have to show the nonvanishing of

$$
\Delta:=\operatorname{det}\left(\begin{array}{ll}
\frac{\partial f_{1}}{\partial t_{1}} & \frac{\partial f_{1}}{\partial t_{2}} \\
\frac{\partial f_{2}}{\partial t_{1}} & \frac{\partial f_{2}}{\partial t_{2}}
\end{array}\right)=\frac{\partial f_{1}}{\partial t_{2}}
$$

at $\left(x_{1}, x_{2}, y_{1}, y_{2}\right)$. From now on we restrict the investigation to particular cases. First, let $n=3$. For $P_{3}(X, Y)$ we have $N=4$ and $M=2$ (cf. Appendix). We now compute $\Delta=\frac{\partial f_{1}}{\partial t_{2}}\left(x_{1}, x_{2}, y_{1}, y_{2}\right)$, where $f_{1}$ is as in (4.5). Setting $y_{2}=x_{1}$, we get

$$
\begin{aligned}
\Delta= & 72 x_{2}\left(x_{2}^{2}+x_{1}^{2}\right)^{3}\left(3440 x_{2}^{2} y_{1}^{4} x_{1}^{10}+7536 y_{1}^{4} x_{2}^{10} x_{1}^{2}-19936 x_{1}^{6} y_{1}^{4} x_{2}^{6}\right. \\
& +34560 x_{1}^{6} y_{1}^{8} x_{2}^{2}-10344 x_{1}^{8} y_{1}^{4} x_{2}^{4}-186624 x_{1}^{2} y_{1}^{12} x_{2}^{2}+920 x_{1}^{4} y_{1}^{4} x_{2}^{8} \\
& +51840 x_{1}^{4} y_{1}^{8} x_{2}^{4}+34560 x_{1}^{2} y_{1}^{8} x_{2}^{6}+8640 y_{1}^{8} x_{2}^{8}-93312 x_{1}^{4} y_{1}^{12}+210 x_{1}^{8} x_{2}^{8} \\
& +168 x_{1}^{6} x_{2}^{10}+84 x_{1}^{4} x_{2}^{12}+168 x_{1}^{10} x_{2}^{6}+84 x_{1}^{12} x_{2}^{4}+24 x_{1}^{14} x_{2}^{2}+744 x_{1}^{12} y_{1}^{4} \\
& \left.+24 x_{1}^{2} x_{2}^{14}-93312 y_{1}^{12} x_{2}^{4}+3 x_{2}^{16}+186624 y_{1}^{16}+8640 x_{1}^{8} y_{1}^{8}+3 x_{1}^{16}-280 y_{1}^{4} x_{2}^{12}\right) .
\end{aligned}
$$

To prove that $\Delta \neq 0$ it suffices to consider the polynomial within the second parentheses, since $72 x_{2}\left(x_{2}^{2}+x_{1}^{2}\right)^{3}$ does not vanish by the algebraic independence 
of $x_{1}$ and $x_{2}$ :

$$
\begin{aligned}
h_{1}\left(x_{1}, x_{2}, y_{1}\right):= & 3440 x_{2}^{2} y_{1}^{4} x_{1}^{10}+7536 y_{1}^{4} x_{2}^{10} x_{1}^{2}-19936 x_{1}^{6} y_{1}^{4} x_{2}^{6} \\
& +34560 x_{1}^{6} y_{1}^{8} x_{2}^{2}-10344 x_{1}^{8} y_{1}^{4} x_{2}^{4}-186624 x_{1}^{2} y_{1}^{12} x_{2}^{2}+920 x_{1}^{4} y_{1}^{4} x_{2}^{8} \\
& +51840 x_{1}^{4} y_{1}^{8} x_{2}^{4}+34560 x_{1}^{2} y_{1}^{8} x_{2}^{6}+8640 y_{1}^{8} x_{2}^{8}-93312 x_{1}^{4} y_{1}^{12} \\
& +210 x_{1}^{8} x_{2}^{8}+168 x_{1}^{6} x_{2}^{10}+84 x_{1}^{4} x_{2}^{12}+168 x_{1}^{10} x_{2}^{6}+84 x_{1}^{12} x_{2}^{4} \\
& +24 x_{1}^{14} x_{2}^{2}+744 x_{1}^{12} y_{1}^{4}+24 x_{1}^{2} x_{2}^{14}-93312 y_{1}^{12} x_{2}^{4}+3 x_{2}^{16} \\
& +186624 y_{1}^{16}+8640 x_{1}^{8} y_{1}^{8}+3 x_{1}^{16}-280 y_{1}^{4} x_{2}^{12} .
\end{aligned}
$$

Let us assume that $\Delta=0$, hence $h_{1}\left(x_{1}, x_{2}, y_{1}\right)=0$. From (4.5) we have $\left(x_{1}^{2}+x_{2}^{2}\right)^{2(N+M)} P_{3}\left(X_{0}, Y_{0}\right)=0$. Using $y_{2}=x_{1}$, it follows that

$$
\begin{aligned}
0= & 9\left(x_{2}^{2}+x_{1}^{2}\right)^{4}\left(-x_{2}^{8}+80 y_{1}^{2} x_{1}^{2} x_{2}^{4}-72 y_{1}^{4} x_{1}^{4}-4 x_{2}^{2} x_{1}^{6}+432 y_{1}^{8}-x_{1}^{8}\right. \\
& -6 x_{2}^{4} x_{1}^{4}-144 y_{1}^{4} x_{1}^{2} x_{2}^{2}+80 x_{1}^{4} y_{1}^{2} x_{2}^{2}-4 x_{2}^{6} x_{1}^{2}-72 y_{1}^{4} x_{2}^{4}-16 y_{1}^{2} x_{2}^{6} \\
& \left.-16 x_{1}^{6} y_{1}^{2}\right)\left(-x_{2}^{8}-80 y_{1}^{2} x_{1}^{2} x_{2}^{4}-72 y_{1}^{4} x_{1}^{4}-4 x_{2}^{2} x_{1}^{6}+432 y_{1}^{8}-x_{1}^{8}-6 x_{2}^{4} x_{1}^{4}\right. \\
& \left.-144 y_{1}^{4} x_{1}^{2} x_{2}^{2}-80 x_{1}^{4} y_{1}^{2} x_{2}^{2}-4 x_{2}^{6} x_{1}^{2}-72 y_{1}^{4} x_{2}^{4}+16 y_{1}^{2} x_{2}^{6}+16 x_{1}^{6} y_{1}^{2}\right) .
\end{aligned}
$$

The algebraic independence of $x_{1}, x_{2}$ over $\mathbb{Q}$ shows that $9\left(x_{2}^{2}+x_{1}^{2}\right)^{4} \neq 0$, hence the number

$$
\begin{aligned}
h_{2}\left(x_{1}, x_{2}, y_{1}\right):= & \left(-x_{2}^{8}+80 y_{1}^{2} x_{1}^{2} x_{2}^{4}-72 y_{1}^{4} x_{1}^{4}-4 x_{2}^{2} x_{1}^{6}+432 y_{1}^{8}-x_{1}^{8}-6 x_{2}^{4} x_{1}^{4}\right. \\
& \left.-144 y_{1}^{4} x_{1}^{2} x_{2}^{2}+80 x_{1}^{4} y_{1}^{2} x_{2}^{2}-4 x_{2}^{6} x_{1}^{2}-72 y_{1}^{4} x_{2}^{4}-16 y_{1}^{2} x_{2}^{6}-16 x_{1}^{6} y_{1}^{2}\right) \\
& \times\left(-x_{2}^{8}-80 y_{1}^{2} x_{1}^{2} x_{2}^{4}-72 y_{1}^{4} x_{1}^{4}-4 x_{2}^{2} x_{1}^{6}+432 y_{1}^{8}-x_{1}^{8}-6 x_{2}^{4} x_{1}^{4}\right. \\
& \left.-144 y_{1}^{4} x_{1}^{2} x_{2}^{2}-80 x_{1}^{4} y_{1}^{2} x_{2}^{2}-4 x_{2}^{6} x_{1}^{2}-72 y_{1}^{4} x_{2}^{4}+16 y_{1}^{2} x_{2}^{6}+16 x_{1}^{6} y_{1}^{2}\right)
\end{aligned}
$$

vanishes. By the assumption $h_{1}=0$ it follows that $\operatorname{Res}_{y_{1}}\left(h_{1}\left(x_{1}, x_{2}, y_{1}\right)\right.$, $\left.h_{2}\left(x_{1}, x_{2}, y_{1}\right)\right)=0$. We obtain

$$
\begin{aligned}
0= & 2^{240} 3^{72} x_{1}^{16} x_{2}^{8}\left(8 x_{1}^{4}+29 x_{2}^{2} x_{1}^{2}+27 x_{2}^{4}\right)^{4}\left(x_{2}-x_{1}\right)^{12}\left(x_{1}+x_{2}\right)^{12} \\
& \times\left(x_{2}^{2}-2 x_{1} x_{2}-x_{1}^{2}\right)^{16}\left(x_{2}^{2}+2 x_{1} x_{2}-x_{1}^{2}\right)^{16}\left(x_{2}^{2}+x_{1}^{2}\right)^{64},
\end{aligned}
$$

a contradiction to the algebraic independence of $x_{1}, x_{2}$ over $\mathbb{Q}$. Thus the AIC proves the algebraic independence of $\vartheta_{3}(6 \tau)$ and $\vartheta_{3}(\tau)$ over $\mathbb{Q}$.

Next, let $n=5$. With $N=6, M=4$, and the polynomial $P_{5}(X, Y)$ listed in the appendix, an analogous computation finally gives the identity

$$
\begin{aligned}
0= & 2^{592} 5^{200} x_{1}^{32} x_{2}^{8}\left(128 x_{1}^{12}-816 x_{1}^{8} x_{2}^{4}+603 x_{1}^{6} x_{2}^{6}+5775 x_{1}^{4} x_{2}^{8}+7569 x_{1}^{2} x_{2}^{10}\right. \\
& \left.+3125 x_{2}^{12}\right)^{4}\left(243 x_{2}^{24}-3580 x_{1}^{2} x_{2}^{22}-315034 x_{1}^{4} x_{2}^{20}+1780 x_{1}^{6} x_{2}^{18}+1040093 x_{1}^{8} x_{2}^{16}\right. \\
& +774920 x_{1}^{10} x_{2}^{14}-2001516 x_{1}^{12} x_{2}^{12}+774920 x_{1}^{14} x_{2}^{10}+1040093 x_{1}^{16} x_{2}^{8} \\
& \left.+1780 x_{1}^{18} x_{2}^{6}-315034 x_{1}^{20} x_{2}^{4}-3580 x_{1}^{22} x_{2}^{2}+243 x_{1}^{24}\right)^{8}\left(x_{1}^{2}-2 x_{1} x_{2}-x_{2}^{2}\right)^{16} \\
& \times\left(x_{1}^{2}+2 x_{1} x_{2}-x_{2}^{2}\right)^{16}\left(x_{1}-x_{2}\right)^{20}\left(x_{1}+x_{2}\right)^{20}\left(x_{1}^{2}+x_{2}^{2}\right)^{96} .
\end{aligned}
$$


The contradiction proves the algebraic independence of $\vartheta_{3}(10 \tau)$ and $\vartheta_{3}(\tau)$ over $\mathbb{Q}$. For the proof of the algebraic independence of $\vartheta_{3}(12 \tau)$ and $\vartheta_{3}(\tau)$ over $\mathbb{Q}$ we have to modify the above formulae. From the double-argument formulae (3.1) we obtain

$$
\begin{aligned}
& \vartheta_{2}^{4}(4 \tau)=\frac{1}{16}\left(\vartheta_{3}-\vartheta_{4}\right)^{4} \\
& \vartheta_{3}^{4}(4 \tau)=\frac{1}{16}\left(\vartheta_{3}+\vartheta_{4}\right)^{4}
\end{aligned}
$$

In Theorem A we replace $\tau$ by $4 \tau$ such that (4.3) holds with

$$
\begin{aligned}
& X_{0}=\frac{n^{2} \vartheta_{3}^{4}(4 n \tau)}{\vartheta_{3}^{4}(4 \tau)}=\frac{16 n^{2} y_{1}^{4}}{\left(y_{2}+x_{2}\right)^{4}}, \\
& Y_{0}=\frac{16 \vartheta_{2}^{4}(4 \tau)}{\vartheta_{3}^{4}(4 \tau)}=\frac{16\left(x_{1}-x_{2}\right)^{4}}{\left(x_{1}+x_{2}\right)^{4}},
\end{aligned}
$$

where $y_{1}=\vartheta_{3}(4 n \tau)$. Finally, we replace $(4.5)$ by

$$
f_{1}\left(t_{1}, t_{2}, u_{1}, u_{2}\right)=\left(t_{2}+u_{2}\right)^{4 N}\left(t_{1}+t_{2}\right)^{4 M} P_{n}\left(\frac{16 n^{2} u_{1}^{4}}{\left(t_{2}+u_{2}\right)^{4}}, \frac{16\left(t_{1}-t_{2}\right)^{4}}{\left(t_{1}+t_{2}\right)^{4}}\right) .
$$

Setting $n=3, N=4, M=2$, and following the above lines of computations, we deduce the following identity:

$$
\begin{aligned}
0= & 2^{376} 3^{72} x_{1}^{8} x_{2}^{8}\left(x_{1}^{4}-12 x_{1}^{3} x_{2}-12 x_{1} x_{2}^{3}+x_{2}^{4}+6 x_{1}^{2} x_{2}^{2}\right)^{16} \\
& \times\left(3 x_{1}^{4}+16 x_{1}^{3} x_{2}+30 x_{1}^{2} x_{2}^{2}+32 x_{1} x_{2}^{3}+27 x_{2}^{4}\right)^{4}\left(x_{1}-x_{2}\right)^{32}\left(x_{1}+x_{2}\right)^{128} .
\end{aligned}
$$

Here the contradiction proves the algebraic independence of $\vartheta_{3}(12 \tau)$ and $\vartheta_{3}(\tau)$ over $\mathbb{Q}$.

Finally, for Theorem 1.1 it remains to prove the algebraic independence of $\vartheta_{3}\left(q^{2^{m}}\right)$ and $\vartheta_{3}(q)$ over $\mathbb{Q}$ for any $m \geqslant 3$. Let $n=2^{m}$. By Theorem 4.1 it suffices to show that the polynomial

$$
\operatorname{Res}_{X}\left(P_{n}(X, Y), \frac{\partial}{\partial Y} P_{n}(X, Y)\right) \in \mathbb{Z}[Y]
$$

does not vanish identically. We know from (3.9) in Lemma 3.2 that

$$
P_{n}(X, Y)=T_{n}\left(X,(1+Y)^{2}, Y\right)=\left(n X-(1+Y)^{2}\right)^{2^{m-2}}+Y U_{n}\left(X,(1+Y)^{2}, Y\right) .
$$

Hence we obtain

$$
\begin{aligned}
P_{n}(X, 0) & =T_{n}(X, 1,0)=\left(2^{m} X-1\right)^{2^{m-2}}, \\
\frac{\partial P_{n}}{\partial Y}(X, 0) & =-2^{m-1}\left(2^{m} X-1\right)^{2^{m-2}-1}+U_{n}(X, 1,0) .
\end{aligned}
$$


On the one hand the polynomial $P_{n}(X, 0)$ in (4.6) has a $2^{m-2}$-fold root $X_{0}$ at $X_{0}=1 / 2^{m}$. On the other hand we know by (4.7) and (3.10) in Lemma 3.2 that

$$
\frac{\partial P_{n}}{\partial Y}\left(X_{0}, 0\right)=U_{n}\left(\frac{1}{n}, 1,0\right)=-2^{2^{m-1}-1} \neq 0 .
$$

This shows that for $Y=0$ the polynomials $P_{n}(X, Y)$ and $\partial P_{n}(X, Y) / \partial Y$ have no common root. Therefore, the resultant of both polynomials with respect to $X$ does not vanish identically. This completes the proof of Theorem 1.1.

\section{Appendix}

The polynomials $P_{3}, P_{5}, P_{7}, P_{9}$, and $P_{11}$ listed below were derived from the proof of Theorem 1.1 in [8].

$$
\begin{aligned}
& P_{3}=9-\left(28-16 Y+Y^{2}\right) X+30 X^{2}-12 X^{3}+X^{4} \text {, } \\
& P_{5}=25-\left(126-832 Y+308 Y^{2}-32 Y^{3}+Y^{4}\right) X+\left(255+1920 Y-120 Y^{2}\right) X^{2} \\
& +\left(-260+320 Y-20 Y^{2}\right) X^{3}+135 X^{4}-30 X^{5}+X^{6}, \\
& P_{7}=49-\left(344-17568 Y+20554 Y^{2}-6528 Y^{3}+844 Y^{4}-48 Y^{5}+Y^{6}\right) X \\
& +\left(1036+156800 Y+88760 Y^{2}-12320 Y^{3}+385 Y^{4}\right) X^{2} \\
& -\left(1736-185024 Y+18732 Y^{2}-896 Y^{3}+28 Y^{4}\right) X^{3} \\
& +\left(1750+31360 Y-1960 Y^{2}\right) X^{4}-\left(1064-2464 Y+154 Y^{2}\right) X^{5} \\
& +364 X^{6}-56 X^{7}+X^{8} \\
& P_{9}=6561-\left(60588-18652032 Y+56033208 Y^{2}-40036032 Y^{3}+11743542 Y^{4}\right. \\
& \left.-1715904 Y^{5}+132516 Y^{6}-5184 Y^{7}+81 Y^{8}\right) X \\
& +\left(250146+427613184 Y+2083563072 Y^{2}+86274432 Y^{3}-57982860 Y^{4}\right. \\
& \left.+4249728 Y^{5}-99288 Y^{6}+576 Y^{7}-9 Y^{8}\right) X^{2} \\
& -\left(607420-1418904064 Y+2511615520 Y^{2}-353755456 Y^{3}+19071754 Y^{4}\right. \\
& \left.-612736 Y^{5}+13960 Y^{6}-64 Y^{7}+Y^{8}\right) X^{3} \\
& +\left(959535+856286208 Y+8468928 Y^{2}-2145024 Y^{3}-808488 Y^{4}\right. \\
& \left.+65664 Y^{5}-1368 Y^{6}\right) X^{4} \\
& -\left(1028952+22899456 Y+1430352 Y^{2}-505152 Y^{3}+38826 Y^{4}\right. \\
& \left.-1728 Y^{5}+36 Y^{6}\right) X^{5} \\
& +\left(757596-13138944 Y+4160448 Y^{2}-417408 Y^{3}+13044 Y^{4}\right) X^{6} \\
& -\left(378072+1138176 Y+16416 Y^{2}-10944 Y^{3}+342 Y^{4}\right) X^{7} \\
& +\left(122895+64512 Y-4032 Y^{2}\right) X^{8}-\left(24060-11136 Y+696 Y^{2}\right) X^{9} \\
& +2466 X^{10}-108 X^{11}+X^{12} \text {, }
\end{aligned}
$$




$$
\begin{aligned}
P_{11}= & 121-\left(1332-2214576 Y+15234219 Y^{2}-21424896 Y^{3}+11848792 Y^{4}\right. \\
& \left.-3309152 Y^{5}+522914 Y^{6}-48896 Y^{7}+2684 Y^{8}-80 Y^{9}+Y^{10}\right) X \\
& +\left(6666+111458688 Y+2532888424 Y^{2}+2367855776 Y^{3}-327773413 Y^{4}\right. \\
& \left.-9982720 Y^{5}+3230480 Y^{6}-161920 Y^{7}+2530 Y^{8}\right) X^{2} \\
& -\left(20020-864654912 Y+12880909668 Y^{2}-5289254784 Y^{3}+744094076 Y^{4}\right. \\
& \left.-43914992 Y^{5}+967461 Y^{6}-2816 Y^{7}+44 Y^{8}\right) X^{3} \\
& +\left(40095+1748954240 Y-175142088 Y^{2}+372281536 Y^{3}-68516998 Y^{4}\right. \\
& \left.+4266240 Y^{5}-88880 Y^{6}\right) X^{4} \\
& -\left(56232-1061669664 Y+132688050 Y^{2}-10724736 Y^{3}+715308 Y^{4}\right. \\
& \left.-28512 Y^{5}+594 Y^{6}\right) X^{5} \\
& +\left(56364+211953280 Y-7454568 Y^{2}-724064 Y^{3}+22627 Y^{4}\right) X^{6} \\
& -\left(40392-24140864 Y+2162116 Y^{2}-81664 Y^{3}+2552 Y^{4}\right) X^{7} \\
& +\left(20295+1448832 Y-90552 Y^{2}\right) X^{8}-\left(6820-36784 Y+2299 Y^{2}\right) X^{9} \\
& +1386 X^{10}-132 X^{11}+X^{12} .
\end{aligned}
$$

The polynomials $P_{2}, P_{4}, P_{8}, P_{16}$, and $P_{32}$ listed below were derived from the proof of Lemma 3.1:

$$
\begin{aligned}
P_{2}= & 2 X-Y^{2}-1 \\
P_{4}= & 4 X-(1+Y)^{2}, \\
P_{8}= & 64 X^{2}-16(1+Y)^{2} X+(1-Y)^{4} \\
P_{16}= & 65536 X^{4}-16384(1+Y)^{2} X^{3}+512\left(3 Y^{4}+4 Y^{3}+18 Y^{2}+4 Y+3\right) X^{2} \\
& -64(1+Y)^{2}\left(Y^{4}+28 Y^{3}+6 Y^{2}+28 Y+1\right) X+(1-Y)^{8}, \\
P_{32}= & 2^{40} X^{8}-2^{38}(1+Y)^{2} X^{7}+2^{32}\left(7 Y^{4}+20 Y^{3}+42 Y^{2}+20 Y+7\right) X^{6} \\
& -2^{28}(1+Y)^{2}\left(7 Y^{4}+164 Y^{3}+42 Y^{2}+164 Y+7\right) X^{5} \\
& +2^{21}\left(35 Y^{8}+552 Y^{7}+2260 Y^{6}+3864 Y^{5}+5010 Y^{4}\right. \\
& \left.+3864 Y^{3}+2260 Y^{2}+552 Y+35\right) X^{4} \\
& -2^{18}(1+Y)^{2}\left(7 Y^{8}+424 Y^{7}+7492 Y^{6}+2968 Y^{5}+15082 Y^{4}\right. \\
& \left.+2968 Y^{3}+7492 Y^{2}+424 Y+7\right) X^{3} \\
& +2^{12}\left(7 Y^{12}-5924 Y^{11}+4174 Y^{10}+33900 Y^{9}+33161 Y^{8}+36536 Y^{7}\right. \\
& \left.+58436 Y^{6}+36536 Y^{5}+33161 Y^{4}+33900 Y^{3}+4174 Y^{2}-5924 Y+7\right) X^{2} \\
& -2^{8}(1+Y)^{2}\left(Y^{12}+660 Y^{11}+15170 Y^{10}+68420 Y^{9}+121327 Y^{8}\right. \\
& +212520 Y^{7}+212380 Y^{6}+212520 Y^{5}+121327 Y^{4}+68420 Y^{3} \\
& \left.+15170 Y^{2}+660 Y+1\right) X+(1-Y)^{16} .
\end{aligned}
$$




\section{References}

[1] B.C. Berndt, Ramanujan's Notebooks, part III, Springer (1991).

[2] C. Elsner, Sh. Shimomura, I. Shiokawa, Algebraic independence results for reciprocal sums of Fibonacci numbers, Acta Arithmetica 148.3 (2011), 205223.

[3] C. Elsner, I. Shiokawa, On algebraic relations for Ramanujan's functions, Ramanujan Journal 29 (2012), 273-294, DOI: 10.1007/s11139-012-9384-8.

[4] A. Hurwitz, R. Courant, Funktionentheorie, 4.Auflage, Springer (1964).

[5] S. Lang, Elliptic Functions, Springer Verlag (1987).

[6] D.F. Lawden, Elliptic Functions and Applications, Springer (1989).

[7] Yu.V. Nesterenko, Modular functions and transcendence questions, Mat. Sb. 187 (1996), 65-96; English transl. Sb. Math. 187, 1319-1348.

[8] Yu.V. Nesterenko, On some identities for theta-constants, Seminar on Mathematical Sciences: Diophantine Analysis and Related Fields, edt. by M. Katsurada, T. Komatsu, and H. Nakada, Keio University, Yokohama, no. 35 (2006), 151-160.

[9] H. Rademacher, Topics in Analytic Number Theory, Die Grundlehren der mathematischen Wissenschaften, Bd.169, Springer, Berlin-Heidelberg-New York (1973).

Address: Carsten Elsner: Fachhochschule für die Wirtschaft, University of Applied Sciences, Freundallee 15, D-30173 Hannover, Germany.

E-mail: carsten.elsner@fhdw.de

Received: 3 April 2013; revised: 22 June 2014 